\title{
Laser shock peening without absorbent coating (LSPwC) effect on 3D surface topography and mechanical properties of 6082-T651 Al alloy
}

\author{
Uroš Trdan , Juan A. Porro , José L. Ocaña Janez Grum
}

\begin{abstract}
A B S T R A C T
The influence of nanosecond laser pulses applied by laser shock peening without absorbent coating (LSPwC) with a Q-switched Nd:YAG laser operating at a wavelength of $\lambda=1064 \mathrm{~nm}$ on $6082-\mathrm{T} 651 \mathrm{Al}$ alloy has been investigated. The first portion of the present study assesses laser shock peening effect at two pulse densities on three-dimensional (3D) surface topography characteristics. In the second part of the study, the peening effect on surface texture orientation and micro-structure modification, i.e. the effect of surface craters due to plasma and shock waves, were investigated in both longitudinal $(\mathrm{L})$ and transverse $(\mathrm{T})$ directions of the laser-beam movement. In the final portion of the study, the changes of mechanical properties were evaluated with a residual stress profile and Vickers micro-hardness through depth variation in the near surface layer, whereas factorial design with a response surface methodology (RSM) was applied. The surface topographic and micro-structural effect of laser shock peening were characterised with optical microscopy, InfiniteFocus $®$ microscopy and scanning electron microscopy (SEM). Residual stress evaluation based on a hole-drilling integral method confirmed higher compression at the near surface layer $(33 \mu \mathrm{m})$ in the transverse direction $\left(\sigma_{\min }\right)$ of laser-beam movement, i.e. $-407 \pm 81 \mathrm{MPa}$ and $-346 \pm 124 \mathrm{MPa}$, after 900 and $2500 \mathrm{pulses} / \mathrm{cm}^{2}$, respectively. Moreover, RSM analysis of micro-hardness through depth distribution confirmed an increase at both pulse densities, whereas LSPwC-generated shock waves showed the impact effect of up to $800 \mu \mathrm{m}$ below the surface. Furthermore, ANOVA results confirmed the insignificant influence of LSPwC treatment direction on micro-hardness distribution indicating essentially homogeneous conditions, in both $L$ and $T$ directions.
\end{abstract}

\section{Introduction}

To reduce surface damage on the parts exposed to demanding conditions, an adequate surface treatment and protection is essential to prolong a service life. Laser shock peening (LSP) is an innovative surface treatment that has already been widely approved as a substitute of conventional Shot Peening (SP).

The basic theoretical idea of laser-driven shock waves was first recognised and explored by the early 1960 s by Fairand and Clauer $[1,2]$. Initially, LSP was developed for the aeronautic industry as the method for the improvement of resistance to fatigue cracking [3-5].

In practice two different LSP processes are known [6]; first (also first discovered) uses protective coating or absorbent layer in order to prevent the material surface from melting or being damaged. In this case high energy laser pulses $(\mathrm{E}<100 \mathrm{~J}$ ) and pulse duration up to $100 \mathrm{~ns}$ are applied. The coating is usually formed with a black paint or Al foil prior to laser irradiation, and the remaining coating is removed after the treatment [7]. The other called laser shock peening without coating (LSPwC) was discover in 1995 [8]. In this process lasers with lower energies (order of a few Joules or less), shorter pulse duration, smaller laser spots, higher coverage and no coating are used [9].

In general both LSP processes require a unique high power density, high repetition laser, which enables plasma generation at the moment of the interaction of laser light and propagation of shock impact waves in the material $[10,11]$. The main effect of LSP is considerable merging, i.e. fusion of dislocations and generation of compressive residual stresses.

Schematic presentation of the LSPWC process is shown in Fig. 1. The principle of compressive residual stress generation by LSPWC was described by Sano et al. [7]; when metallic material without protective coating is irradiated underwater by laser pulses of specific characteristics the surface instantaneously evaporates through the ablative interaction. Water confines the evaporating material, which is immediately ionized and forms plasma by inverse braking radiation (bremsstrahlung). Energy absorption in the plasma generates a dominant shock wave on the material with an intensity of several GPa which creates a permanent strain. Owing to low energy of laser pulses 


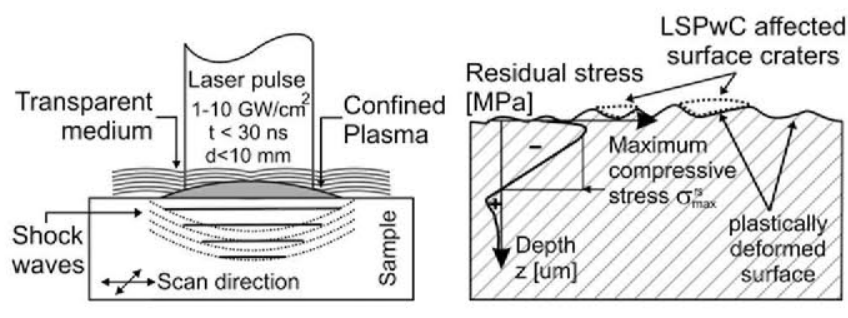

Fig. 1. Laser shock peening (LSPwC) principle.

and short pulse duration the thermal damage is very small or even negligible. Moreover, practically no thermal affected depth is present due to exceedingly local surface ablation - effect of shock waves is prevailing.

The shocked surface after LSPWC is plastically deformed and wants to stretch, whereas the surrounding elastically responding material below the impact restrains stretching. As a result, high compressive residual stresses are generated and confined to a shallow surface layer whilst balancing low-tensile residual stresses are spread deeper throughout the cross-section of the material [12].

Enhancement of fatigue, corrosion, stress corrosion and wear resistance has already been proved for various types of materials, such as aluminium and titanium alloys and different types of steel [11-18]. In our recent publication [18], we investigated the effect of LSPwC treatment on corrosion behaviour on an AA6082 aluminium alloy in a $0.6 \mathrm{M} \mathrm{NaCl}$ solution. Both cyclic polarisation (CP) and electrochemical impedance spectroscopy (EIS) measurements confirmed the beneficial effect of LSPWC treatment. CP results showed enhanced passivity with corrosion current reduction by as much as a factor of 12 , compared to the untreated specimen. Moreover, EIS after $24 \mathrm{~h}$ confirmed an almost seven times higher polarisation resistance after LSPWC, compared to the untreated specimen, i.e. $45 \mathrm{k} \Omega \mathrm{cm}^{2}$ and $6.7 \mathrm{k} \Omega \mathrm{cm}^{2}$, respectively. The results were shown to be attributable to the modification of surface amorphous $\mathrm{Al}_{2} \mathrm{O}_{3}$ film into more stable oxide form, such as $\alpha-\mathrm{Al}_{2} \mathrm{O}_{3}$ or $\mathrm{Al}_{2} \mathrm{O}_{3}$-sapphire, with a binding energy of $75.1 \mathrm{eV}$ and to the development of residual stresses after LSPwC.

Aluminium alloys represent a very important category of materials, which have been widely used in the aircraft, automotive, marine and construction industries, due to their low cost, good weight/ strength ratio and high corrosion resistance $[19,20]$.

Although, several studies $[3,11,15,21]$ have investigated the influence of laser shock peening on aluminium alloys' topographical characteristics, there is still insufficient information, especially due to the complexity of the LSP process.

Recently, Dai et al. [22] investigated LSP's effect on aeronautical LY2 Al alloy with different initial surface conditions. It was reported that one LSP impact on the surface roughness of LY2 Al alloy will tend to be stable; however, after multiple LSP impacts, surface height distribution scattered from $-2.5 \mu \mathrm{m}$ to $2.5 \mu \mathrm{m}$. Moreover, it was found that after three LSP impacts, the value of saturated compressive residual stress is independent of surface roughness. In another study, Lu et al. [23] reported obvious micro-structure refinement of LY2 Al alloy due to the ultra-high plastic strain induced by multiple LSP impacts, whereas the minimum grain size in the top surface after multiple LSP impacts was about 100-200 nm.

In practice, 3D topography measurement possesses several advantages over the more commonly accepted profilometry methods [24-27]. Three-dimensional topography includes more realistic surface characterisation with a reduction of error, and can provide the actual bearing area, material and void volumes texture direction and texture type information $[24,27,28]$.

This study is focused on the practical application of the threedimensional measurements of the InfiniteFocus ${ }^{\circledR}$ (Focus-Variation) device on laser shock-peened aluminium alloy, aiming to characterise the LSPWC effect on 3D amplitude and spatial topographic parameters.
Moreover, surface texture orientation with the auto-correlation function was investigated. Furthermore, investigation of the LSPwC effect on mechanical properties by means of micro-hardness and residual stress distribution was evaluated.

\section{Materials and methods}

\subsection{Base material}

The material tested was a 6082-T651 aluminium alloy supplied as a drawn round bar of $\emptyset 40 \mathrm{~mm}$ with composition in mass \% as Si: 0.98 , Mg: 0.67, Mn: 0.53, Fe: 0.25, Ti: 0.03, some minor elements ( $\mathrm{Cr}, \mathrm{Zn}$, Ti, $\mathrm{Cu}$ ) and the remaining was $\mathrm{Al}$.

The SEM microstructure of the material in the initial condition with corresponding EDX analysis of region "1" is shown in Fig. 2. Microstructure analysis was carried out with a JEOL JXA-8600M scanning electron microscope. As the etchant, Keller's reagent was used (94 $\mathrm{mL}$ water $+3 \mathrm{~mL}$ nitric acid $+2 \mathrm{~mL}$ hydrochloric acid $+1 \mathrm{~mL}$ hydrofluoric acid).

Microstructure analysis revealed a basic aluminium matrix in which fine inter-metallic precipitates, i.e. second-phases are homogeneously distributed, which contribute to higher material hardness and strength. The size of the larger precipitates was approximately $4 \mu \mathrm{m}$ (point 1), whereas the size of the smaller precipitates was between $0.5 \mu \mathrm{m}$ and $1.0 \mu \mathrm{m}$ (point 3,2 ), respectively. EDX analysis results of the precipitates and mechanical properties of AA6082 alloy are given in Tables 1 and 2, respectively. Material in the base condition confirmed three chemical compounds of second phase inter-metallic phases. First, the largest inter-metallic phase (point 1) consisted of $\mathrm{Al}_{\mathrm{x}}(\mathrm{Si}, \mathrm{Mn}, \mathrm{Fe})$, the second one (point 2) consisted of $\mathrm{Al}_{\mathrm{x}}(\mathrm{Zn}, \mathrm{Mn}, \mathrm{Fe}, \mathrm{Cu})$ and the smallest inter-metallic phase (point 3) of $\mathrm{Al}_{\mathrm{x}}(\mathrm{Mn}, \mathrm{Fe})$.

\subsection{Laser shock peening}

LSP was carried out using the method of closed ablation without protective coating (LSPwC), where water served as the confining medium. LSPWC surface treatment was performed using a Quanta-Ray Q-switched Nd:YAG laser with a wavelength of $1064 \mathrm{~nm}$, operating at $10 \mathrm{~Hz}$ repetition rate. Full width at half maximum (FWHM) of the pulses was $10 \mathrm{~ns}$ and the spot diameter was $1.5 \mathrm{~mm}$. In the experiments laser beam had a "quasii" Gaussian shape profile, whereas beam divergence of output was $\theta_{f} \leq<0.5 \mathrm{mrad}$ (full angle measured at FWHM points), with beam pointing stability less than \pm 50 urad.

The maximum pulse energy was $1.9 \mathrm{~J} / \mathrm{cm}^{2}$, thus peak power density $\left(Q_{p}=E_{p} /(A \tau)\right)$ was equal to $10.75 \mathrm{GW} / \mathrm{cm}^{2}$. Here $A$ represents the beam spot area $\left(A=\pi D^{2} / 4\right)$ and $\tau$ is the duration of the laser pulse at FWHM. Flat mirrors and a convergent lens $(f=200 \mathrm{~mm}$, bi-convex $)$

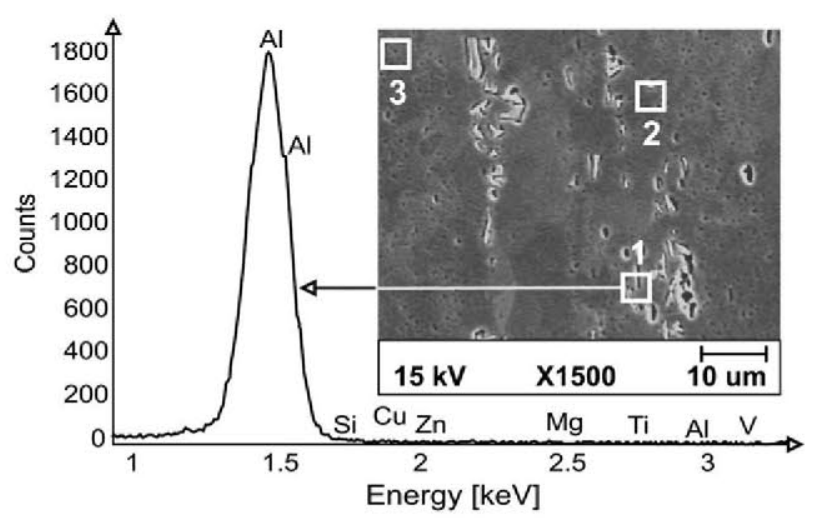

Fig. 2. SEM morphology of the base/untreated material with EDX analysis of the large intermetallic phase (point 1). 
Table 1

EDX element analysis of base material second-phase particles.

\begin{tabular}{|c|c|c|c|c|c|c|}
\hline \multirow[t]{3}{*}{ El. } & \multicolumn{3}{|l|}{ K-ratio } & \multicolumn{3}{|c|}{ Atomic [\%]/element wt. [\%] } \\
\hline & Area & Area & Area & Area & Area & Area \\
\hline & 1 & 2 & 3 & 1 & 2 & 3 \\
\hline $\mathrm{Si}$ & 0.0026 & l & / & $0.53 / 0.55$ & / & / \\
\hline $\mathrm{Mg}$ & / & / & / & / & / & 1 \\
\hline $\mathrm{Mn}$ & 0.0039 & 0.0042 & 0.0030 & $0.23 / 0.46$ & $0.24 / 0.49$ & $0.18 / 0.36$ \\
\hline $\mathrm{Fe}$ & 0.0018 & 0.0036 & 0.0021 & $0.10 / 0.21$ & $0.20 / 0.42$ & $0.12 / 0.24$ \\
\hline $\mathrm{Zn}$ & 1 & 0.0051 & I & / & $0.26 / 0.63$ & 1 \\
\hline $\mathrm{Cu}$ & I & 0.0027 & 1 & 1 & $0.14 / 0.32$ & 1 \\
\hline $\mathrm{Al}$ & 0.9821 & 0.9622 & 0.9890 & $99.14 / 98.78$ & $99.15 / 98.14$ & $99.71 / 99.4$ \\
\hline Total & 0.9904 & 0.9778 & 0.9941 & $100 / 100$ & $100 / 100$ & $100 / 100$ \\
\hline
\end{tabular}

Table 2

Mechanical properties of 6082-T651 Al alloy.

\begin{tabular}{lr}
\hline Property & \multicolumn{1}{c}{ Value } \\
\hline Tensile strength $-R_{m}\left[\mathrm{~N} / \mathrm{mm}^{2}\right]$ & 348 \\
Yield stregth $-R_{p 0.2}\left[\mathrm{~N} / \mathrm{mm}^{2}\right]$ & 320 \\
Modulus of elasticity $-E\left[\mathrm{~N} / \mathrm{mm}^{2}\right]$ & 70000 \\
Modulus of rigidity $-G\left[\mathrm{~N} / \mathrm{mm}^{2}\right]$ & 26400 \\
Vickers hardness $-H V$ & 92 \\
Density $-\rho\left[\mathrm{kg} / \mathrm{m}^{3}\right]$ & 2710 \\
\hline
\end{tabular}

are used to deliver the pulse produced by the laser. Both optical components are AR-coated, which guarantees high transmittance efficiency [29].

In irradiation setup, we have used purified water as confining medium in order to avoid the formation of water bubbles or the concentration of impurities coming from the material ablation due to laser treatment. The length of the water column (confining medium) was about $4 \mathrm{~cm}$. The appearance of suspended elements can affect the LSP process by their interaction with the high energy laser beam.

Two pulse densities, i.e. of 900 and 2500 pulses $/ \mathrm{cm}^{2}$ were selected and controlled with a movable $2 \mathrm{D}$ computer-aided $\mathrm{x}-\mathrm{y}$ table, i.e. controlling the velocity of the system; the desired pulse density was obtained. LSPwC treatment was performed on $\emptyset 40 \mathrm{~mm} \times 8 \mathrm{~mm}$ thick discs (made from extruded rod), where the treated area was equal to $20 \mathrm{~mm} \times 20 \mathrm{~mm}$. Schematic presentation of the specimentreated area and the LSPwC surface scan is given in Fig. 3.

\subsection{Surface characterisation}

The examination of the LSPWC homogeneity and determination of LSPWC effect on the selected three-dimensional (3D) amplitude parameters was performed with a non-contact Alicona G4 3D optical InfiniteFocus ${ }^{\circledR}$ Measuring (IFM) device. The operating principle is based on Focus-Variation, which combines the small depth of focus of an optical system with vertical scanning. This technique enables high-resolution measurements of surfaces with strongly varying roughness with steep flanks up to $80^{\circ}$ [28,30-32]. The chosen magnification was $20 \times$, with 7 million captured points, each point size being $438 \mathrm{~nm}$, with a unified cut-off wavelength $\lambda_{c}=200 \mu \mathrm{m}$. The selected analysed area was $1320 \mu \mathrm{m} \times 980 \mu \mathrm{m}$, with optical lateral resolution of $800 \mathrm{~nm}$ and a vertical resolution of $100 \mathrm{~nm}$. The selection of both, vertical and lateral resolutions can be realized through a simple change of objectives [30]. In focus variation technique vertical resolution of a measurement is the smallest height step (z-axis) that can be measured, whereas lateral resolution is the distance from one measurement point to the next measurement point on the specimen surface (xy-plane). In Focus-Variation measurement the distance between the object and objective lens is varied and images are continuously captured. As the result IFM G4 system can measure 3D datasets which includes form, waviness and roughness of analysed specimen [30].

Afterwards, the effect of the LSPwC on the micro-structure and induced surface craters in both longitudinal and transverse directions of laser-beam movement was characterised with optical microscopy (OM) using Meiji MT-7100 for microscopic analysis and Olympus SZX 10 for macroscopic analysis of surface topography condition. In addition, the areas of the LSPWC surface craters were analysed using UTHSCSA ImageTool Version 3.0 (IT), which enabled determination of real area inside the specific LSP-induced surface crater. The area of the LSPWC-induced craters due to surface ablation and consecutive shock waves was determined in a cross section view as the real area between two neighbouring peaks. Measurements of surface crater areas were conducted on at least three different locations for each condition of LSPWC process.

\subsection{Micro-hardness and residual stress}

The micro-hardness through depth variation prior to and after LSPWC was measured using the Vickers method. Micro-hardness measurements were made with $200 \mathrm{~g}$ load and $20 \mathrm{~s}$ load time. In order to determine the LSPWC effect on micro-hardness distribution, design of experiment (DOE) with response surface methodology (RSM) was applied. DOE is commonly required if meaningful conclusions about the process have to be extracted from the measured responses, which provides information on particular parameter effects, their interaction effects and the statistical significance of regression models [33]. DOE results were obtained using a DesignExpert software program.

Residual stress profiles were measured in accordance with ASTM standard [34], where strain gages CEA-06-062-UM along with RS-200 Milling Guide, Vishay Measurement Group were used. Strains measurement were performed by incremental hole drilling measurements, increment being $0.1 \mathrm{~mm}$, whereas 11 measurements were performed, thus a depth of $1.1 \mathrm{~mm}$ for a single measurement was achieved. In order to obtain reliability of the measurements three and two separate measurements were performed on LSPWC and untreated specimens, respectively.

The final residual-stress (RS) profile in the LSPwC specimens was determined by the integral method, using $\mathrm{H}$-drill version 3.10 software [35]. This method enables low sensitivity to experimental errors and high resolution of calculated residual stresses. Despite usage incremental drilling of $0.1 \mathrm{~mm}$ and the first strain measurement obtained at this depth, the integral method provides first RS data on
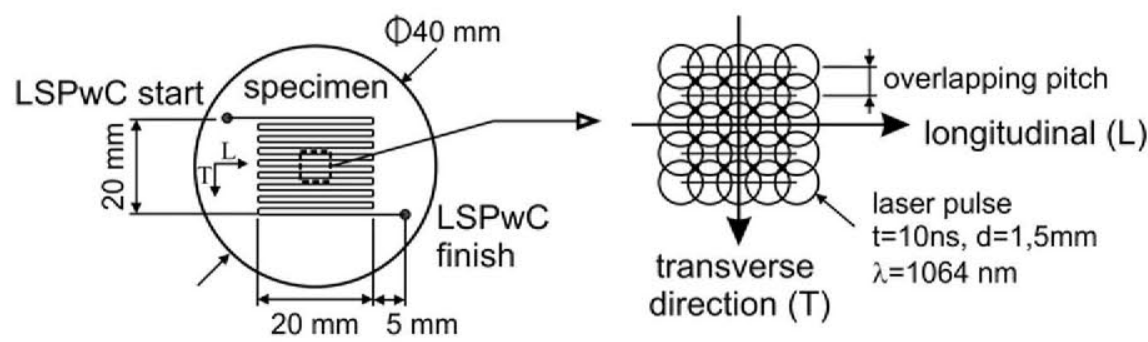

Fig. 3. Illustration of LSPWC-treated area. 
Table 3

Surface 3D amplitude parameters of various samples.

\begin{tabular}{|c|c|c|c|c|}
\hline Parameter & Initial condition/no LSP & LSP - 900 pulses $/ \mathrm{cm}^{2}$ & LSP -2500 pulses $/ \mathrm{cm}^{2}$ & Description \\
\hline$S_{a}[\mu \mathrm{m}]$ & 0.520 & 2.392 & 4.123 & Average height of selected area \\
\hline$S_{q}[\mu \mathrm{m}]$ & 0.653 & 3.039 & 5.115 & Root-mean-square height of selected area \\
\hline$S_{p}[\mu \mathrm{m}]$ & 2.009 & 8.134 & 16.955 & Maximum peak height of selected area \\
\hline$S_{v}[\mu \mathrm{m}]$ & 3.316 & 8.483 & 16.795 & Maximum valley depth of selected area \\
\hline$S_{z}[\mu \mathrm{m}]$ & 5.325 & 16.617 & 33.750 & Maximum height of selected area \\
\hline$S_{10 z}[\mu \mathrm{m}]$ & 4.864 & 15.406 & 25.840 & Ten point height of selected area \\
\hline$S_{s k}[/]$ & -0.387 & -0.054 & -0.083 & Skewness of selected area \\
\hline$S_{k u}[/]$ & 3.395 & 2.491 & 2.819 & Kurtosis of selected area \\
\hline$S_{d q}[/]$ & 0.141 & 0.288 & 0.486 & Root mean square gradient \\
\hline
\end{tabular}

a specific depth of $33 \mu \mathrm{m}$. Nevertheless, this method is the right choice when measuring rapidly varying residual stresses and provides a separate evaluation of residual stress within each increment of depth used during the hole-drilling measurements [36]. However, the sensitivity of the calculated stresses is also the most severe.

Therefore, according to $\mathrm{H}$-drill recommendation automatic smoothing was chosen, with the Tikhonov regularization scheme to enhance stability and resistance to experimental errors, stabilization of the Integral Method stress calculation [35].

\section{Results and discussion}

\subsection{Surface characterisation}

Surface 3D amplitude parameters for different specimens are shown in Table 3 . As can be seen from the results, pulse density indeed has a significant effect on the final topography surface roughness, where the untreated specimen shows the lowest roughness values. For example, the 3D roughness $S_{a}$, evaluated over the complete measured surface, achieved the minimum value of $0.520 \mu \mathrm{m}$ for the untreated specimen; the LSPwC-specimen treated with 900 pulses $/ \mathrm{cm}^{2}$ achieved $S_{a}=2.392 \mu \mathrm{m}$ and LSPWC specimen treated with 2500 pulses $/ \mathrm{cm}^{2}$, achieved about $72 \%$ larger $S_{a}$ value compared to 900 pulses $/ \mathrm{cm}^{2}$, i.e. $4.123 \mu \mathrm{m}$.

Fig. 4 shows the auto-correlation model of the specimens, which was applied in order to determine the LSPWC effect on surface texture direction. The auto-correlation function enables useful information about the texture character of the surface; it also suggests that the surface of the non-treated specimen after cutting reveals a strong tendency of the surface texture orientation in the cutting direction. Meanwhile, specimens after LSPwC show a very small tendency of the surface texture orientation, although it is more pronounced with a higher pulse density level.

For further surface texture information, spatial parameters, i.e. $S_{a l}$ (the fastest decay auto-correlation length) and $S_{t r}$ (texture aspect ratio) was applied. $S_{a l}$ is defined as the shortest horizontal distance of the $A A C F$ (auto-correlation function), which has the fastest decays to 0.2 in any direction $[30,36]$. Parameter $S_{t r}$ is the parameter used to identify the surface texture pattern (isotropy vs. anisotropy) [30,37]. It is defined as the ratio of the fastest to the slowest decay auto-correlation lengths [25]. Parameters $S_{a l}$ and $S_{t r}$ are given by the following equation:

$S_{a l}=\min \left(\sqrt{\tau_{x}^{2}+\tau_{y}^{2}}\right)$

$S_{t r}=\frac{\min \left(\sqrt{\tau_{x}^{2}+\tau_{y}^{2}}\right)}{\max \left(\sqrt{\tau_{x}^{2}+\tau_{y}^{2}}\right)}, 0<S_{t r} \leq 1$

$R\left\{\left(\tau_{x}, \tau_{y}\right): \operatorname{AACF}\left(\tau_{x}, \tau_{y}\right) \leq 0.2\right\}$

where $\tau_{x}$ and $\tau_{y}$ are the lag distances in the $x$ and $y$ directions; $R\left(\tau_{x}, \tau_{y}\right)$ is the auto-correlation function.

Larger value of $S_{t r}$ (towards 1 ) indicates isotropic surface, whilst a smaller value of $S_{t r}$ (towards 0 ) indicates anisotropy with significant texture.

The results of the analysis given in Table 4 confirmed a significant difference between the treatment conditions. The highest autocorrelation length parameter- $S_{a l}$ was calculated after treatment with 2500 pulses $/ \mathrm{cm}^{2}$ (30.981 $\left.\mu \mathrm{m}\right)$, and the lowest $S_{a l}$ value of $8.763 \mu \mathrm{m}$ was calculated for the untreated specimen. The results indicate that the surface of the untreated specimen is dominated by higher frequency components compared to the laser-peened specimens, which are dominated by low frequencies. The large value of surface texture aspect ratio $\left(S_{t r}\right)$ after LSPwC indicates uniform texture in all directions i.e., there is no defined lay.

In order to evaluate the influence of LSPWC on the surface condition even further, surface craters due to plasma and shock waves effect were investigated in both longitudinal $(L)$ and transverse $(T)$ directions of laser-beam movement, i.e. scan direction.

From the micro-section comparison in both $L$ and $T$ directions of the LSPWC passage (Fig. 5), it can be seen that specimen treated with 900 pulses $/ \mathrm{cm}^{2}$ shows similar surface condition in both directions.
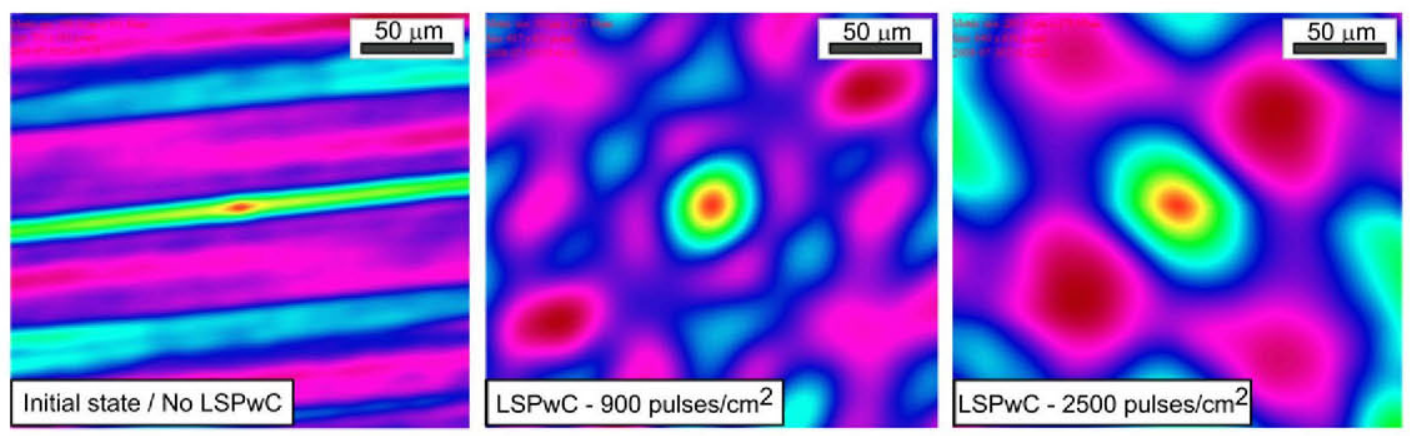

Fig. 4. Auto-correlation surface roughness model. 
Table 4

Spatial parameters results.

\begin{tabular}{llll}
\hline Parameter & No LSP & $900 \mathrm{pulses} / \mathrm{cm}^{2}$ & $2500 \mathrm{pulses} / \mathrm{cm}^{2}$ \\
\hline$S_{a l}[\mu \mathrm{m}]$ & 8.763 & 24.668 & 30.981 \\
$S_{t r}[/]$ & 0.056 & 0.640 & 0.792 \\
\hline
\end{tabular}

The calculated crater depth (peak-to-peak amplitude) after LSPwC treatment with 900 pulses $/ \mathrm{cm}^{2}$ is in the range of $(12 \pm 4.24) \mu \mathrm{m}$ in $L$ direction and of $(18.5 \pm 2.12) \mu \mathrm{m}$ in $T$ direction, respectively. However, after LSPwC treatment with 2500 pulses $/ \mathrm{cm}^{2}$, the surface condition in the transverse $(T)$ direction shows a higher increase of the LSPWCinduced crater depth. In the $L$ direction, the crater depth is in the range of $(19 \pm 2.12) \mu \mathrm{m}$ in $T$ direction much larger peak-to-peak amplitude and wave period of the LSPWC-induced craters are observed. In this case, crater depth, i.e. peak-to-peak amplitude is in the range of $(45.5 \pm 7.78) \mu \mathrm{m}$. Moreover, the wave period is measured to $293.7 \mu \mathrm{m}$, which is by the factor 4.3 larger compared to LSPwC specimen treated with 900 pulses $/ \mathrm{cm}^{2}$, where wave period of $66.8 \mu \mathrm{m}$ was obtained.

Fig. 6 shows the results of crater area after LSPWC, which was measured on three different locations on each specimen and further analysed using ImageTool $(B)$ software. As demonstrated in Fig. 5, the crater area after LSPwC treatment with 900 pulses $/ \mathrm{cm}^{2}$ indicate values of $867.92{\mu \mathrm{m}^{2}}^{2}$ in the longitudinal direction, whereas in the transverse direction, the surface exhibited a crater area value of $1088.12 \mu \mathrm{m}^{2}$, which is about $220 \mathrm{\mu m}^{2}$ larger than in $L$ direction. In the case of higher pulse density, an increase of the crater area in the $T$ direction compared to the $L$ direction is about $251 \mu \mathrm{m}^{2}$, whereas in comparison with $900 \mathrm{pulses} / \mathrm{cm}^{2}$ specimen in the $L$ direction, an increase of $470 \mu \mathrm{m}^{2}$ is observed.

Standard deviation was almost equal for all specimens in the range $7.35 \mu \mathrm{m}^{2}-7.87 \mu \mathrm{m}^{2}$. Changes in the crater area due to different pulse densities are highly congruent with non-dimensional 3D surface parameters, i.e. $S_{k u}$ and $S_{d q}$ (Table 3). Note that the kurtosis of selected area $-S_{k u}$, which describes the expansion and the distribution of heights, is lower for the specimen treated 900 pulses $/ \mathrm{cm}^{2}$ compared to the specimen treated with higher pulse density. This indicates that the majority of the heights are in a smaller range of height compared to the other LSPwC specimen. Furthermore, root mean square gradient $-\mathrm{S}_{d q}$, describing the mean slope of the surface texture also increases with higher pulse density. Value of 0.486 for LSPwC with 2500 pulses $/ \mathrm{cm}^{2}$ indicates that the surface is composed of steep surface parts, whereas $S_{d q}=0.141$ for the untreated specimen indicates that the surface consist mainly of flat surface parts. The theory of non-dimensional 3D surface parameters is detailed in Ref. [30].

Furthermore, results obtained in our experiments are in accordance with the expectations after strain hardening of soft materials $[3,11,15,16]$.

\subsection{Micro-hardness and residual stresses}

For analysis of the effect of LSPwC scan direction and pulse density on micro-hardness, the design of experiment-DOE (factorial design)

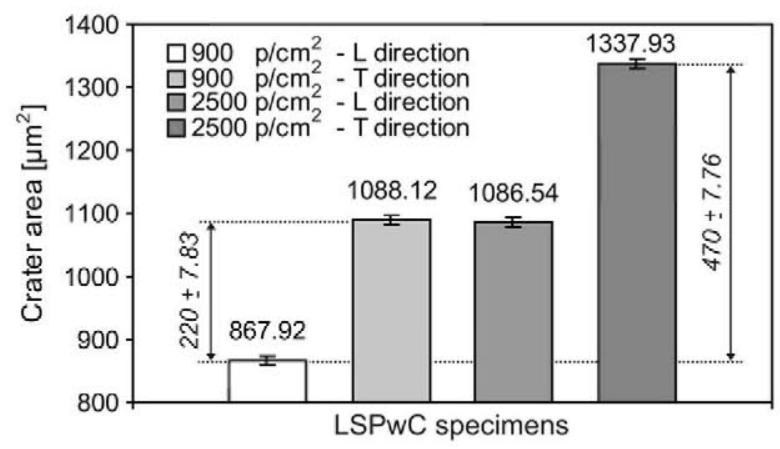

Fig. 6. Surface crater areas after LSPwC.

with response surface methodology (RSM) aided with analysis of variance (ANOVA) was applied. Factorial design is particularly useful when an influence of at least two factors to an output response of the experimental process is treated [38,39].

Fig. 7 shows the response surface and contour plot diagram of micro-hardness in the longitudinal and transverse directions of the LSPwC surface scan, respectively. Numerals in the white boxes in contour plot diagram represent microhardness calculated values, whereas red and pink points in the response surface diagram represent design points above and below predictions, respectively.

The final equation of response surface polynomial in terms of coded factors obtained is:

$H V=+108.95+5.25 A-3.40 B-0.27 C-3.83 A B$

$-1.06 A C-0.51 B C-12.18 A^{2}+1.65 B^{2}$

where coded factors in the equation are as follows; $A$ is pulse density level, $B$ represents depth at which the measurement was taken and $C$ is the direction of LSPWC surface scan direction.

From the RSM micro-hardness results, the following conclusions can be made:

- ANOVA results of RSM model confirmed factors $A, B, A B$, and $A^{2}$ as the factors with significant influence on resulting micro-hardness in the near surface layer.

- According to ANOVA, pulse density $(A)$ is the main parameter affecting the micro-hardness distribution.

- Micro-hardness results depend on LSPwC pulse density and the depth at which the measurements are taken.

- ANOVA results presented in Fig. 7 confirmed that direction LSPwC treatment, i.e. surface scan does not have influence on the micro-hardness, although higher strain hardening depth is observed in the $L$ direction.

- Response surface and contour diagrams of micro-hardness (Fig. 7) confirmed that in general higher surface hardening is achieved with a higher pulse density level. The highest microhardness in the near-surface $(50 \mu \mathrm{m})$ was measured at the LSPwC specimen treated with 2500 pulses $/ \mathrm{cm}^{2}$ in $L$ direction, i.e. $112 \mathrm{HV}_{0.2}$. After
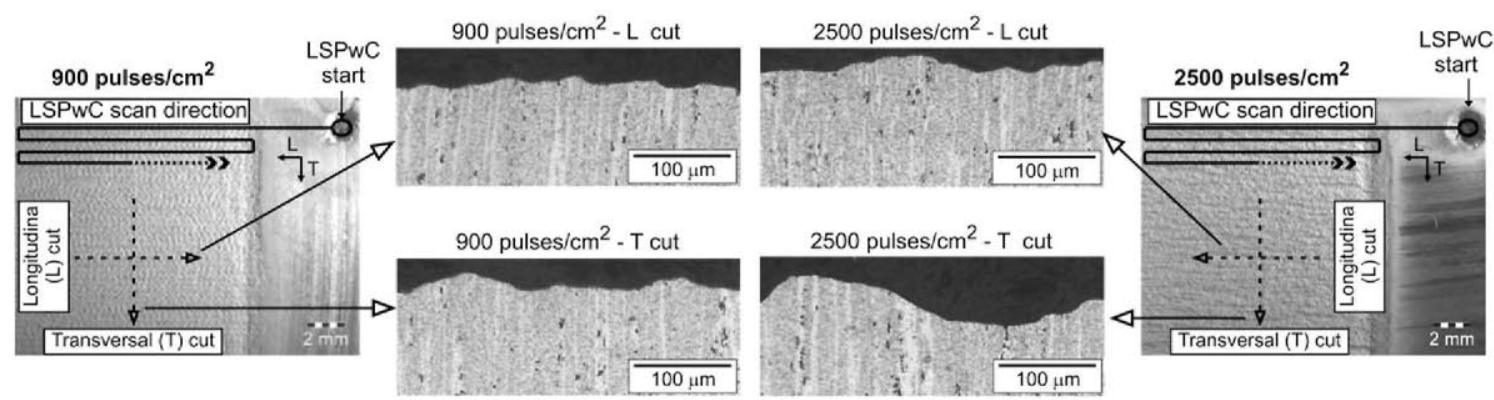

Fig. 5. Schematic presentation of LSPwC surface scan and micro-section of samples treated with different pulse densities. 

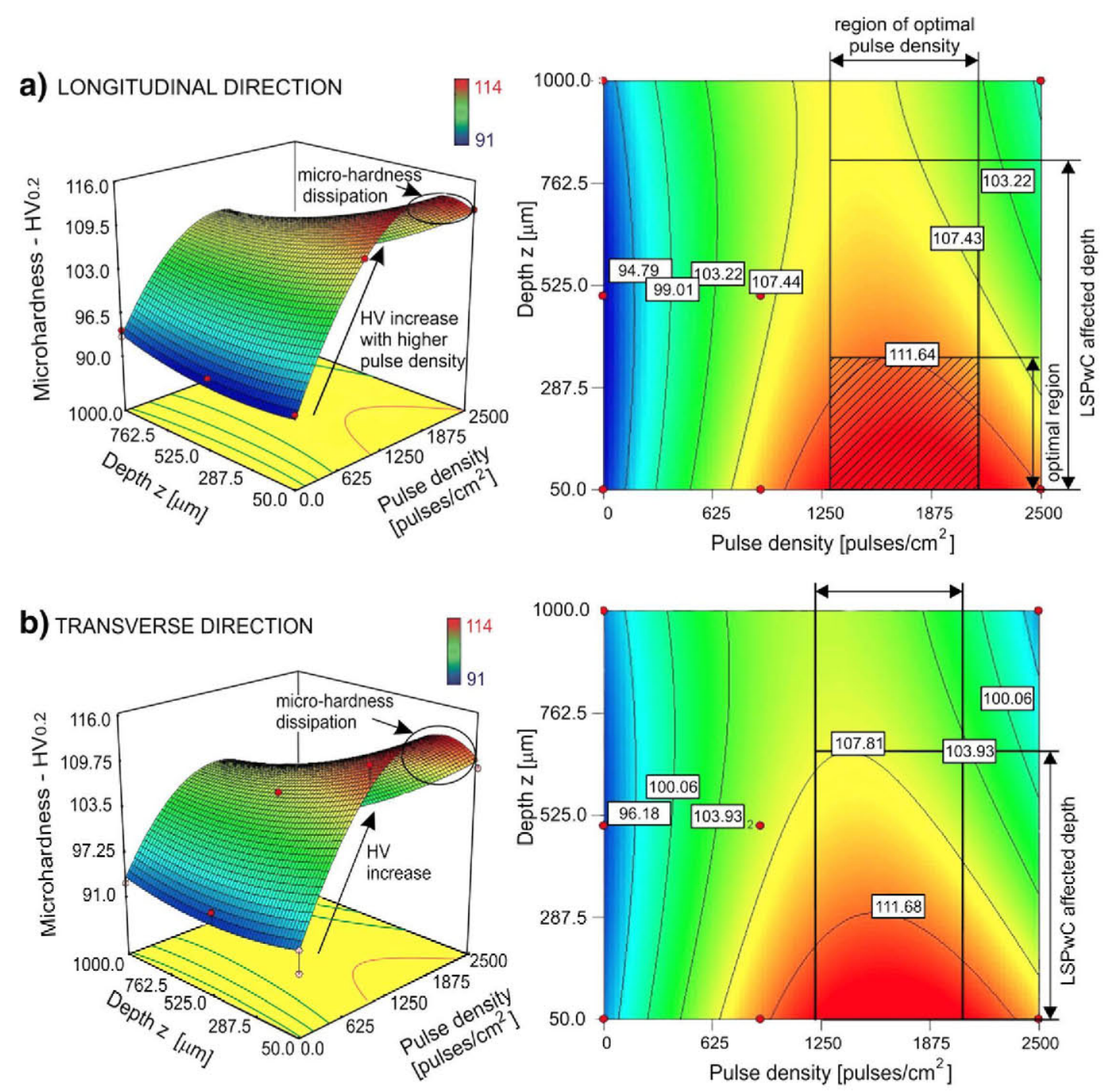

Fig. 7. Response surface and contour plot of micro-hardness in (a) longitudinal and (b) transverse direction of LSPwC surface scan.

900 pulses $/ \mathrm{cm}^{2}$ microhardness of $110 \mathrm{HV}_{0.2}$ was achieved. In comparison to the untreated material $\left(92 \mathrm{HV}_{0.2}\right)$, the microhardness increased by $19.6 \%$ with 900 pulses $/ \mathrm{cm}^{2}$ and $21.7 \%$ with 2500 pulses $/ \mathrm{cm}^{2}$, respectively

- From RSM also the region of optimal pulse density was determined (marked in Fig. 7) as the region with a highest microhardness $\mathrm{HV}_{0.2}$ increase after LSPWC.

- Fig. 8 shows normal probability plot versus studentised residuals, where a $\mathrm{R}^{2}$ value 0.943 of with adequate precision ratio (signal to noise ratio) of 15.344 indicate an adequate signal for navigation of the design space, i.e. satisfies the assumptions of the analysis of variance.

Reduction and dissipation of the micro-hardness values are probably associated with increase of thermal effect due to higher pulse density [40]. Therefore, evaluating the possible relaxation of strain hardening at the surface analysis of residual stress profile is essential.

The distribution of principal residual stresses as a function of depth is shown in Fig. 9. The principal residual stresses $\sigma_{\max }$ and $\sigma_{\min }$ represent the stress component in the longitudinal (parallel) and transverse (perpendicular) of the LSPWC-scan direction, respectively. The residual stresses in the initial state specimen are about the same in both longitudinal and transverse directions, and they amount from $+47 \pm$ $15 \mathrm{MPa}$ to $-21 \pm 7 \mathrm{MPa}$. Such results confirm an adequate T-651 temper state for initial material condition.

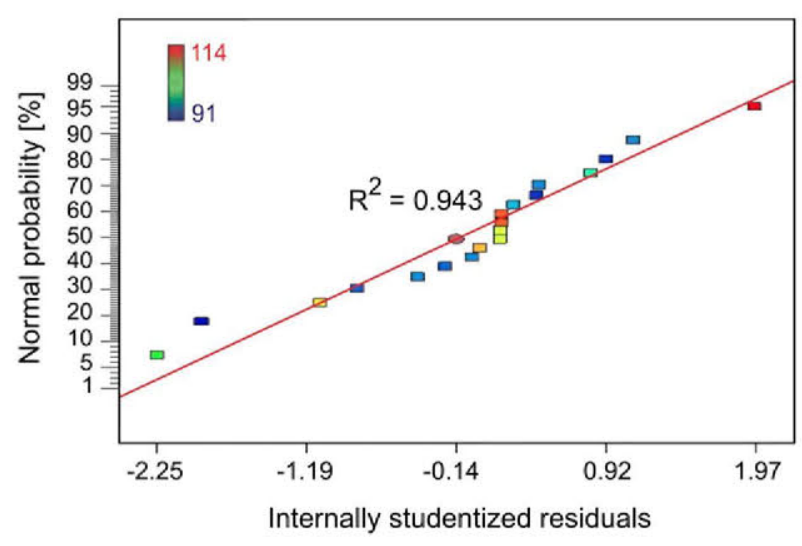

Fig. 8. Normal plot of probability (\%) versus studentised residuals. 

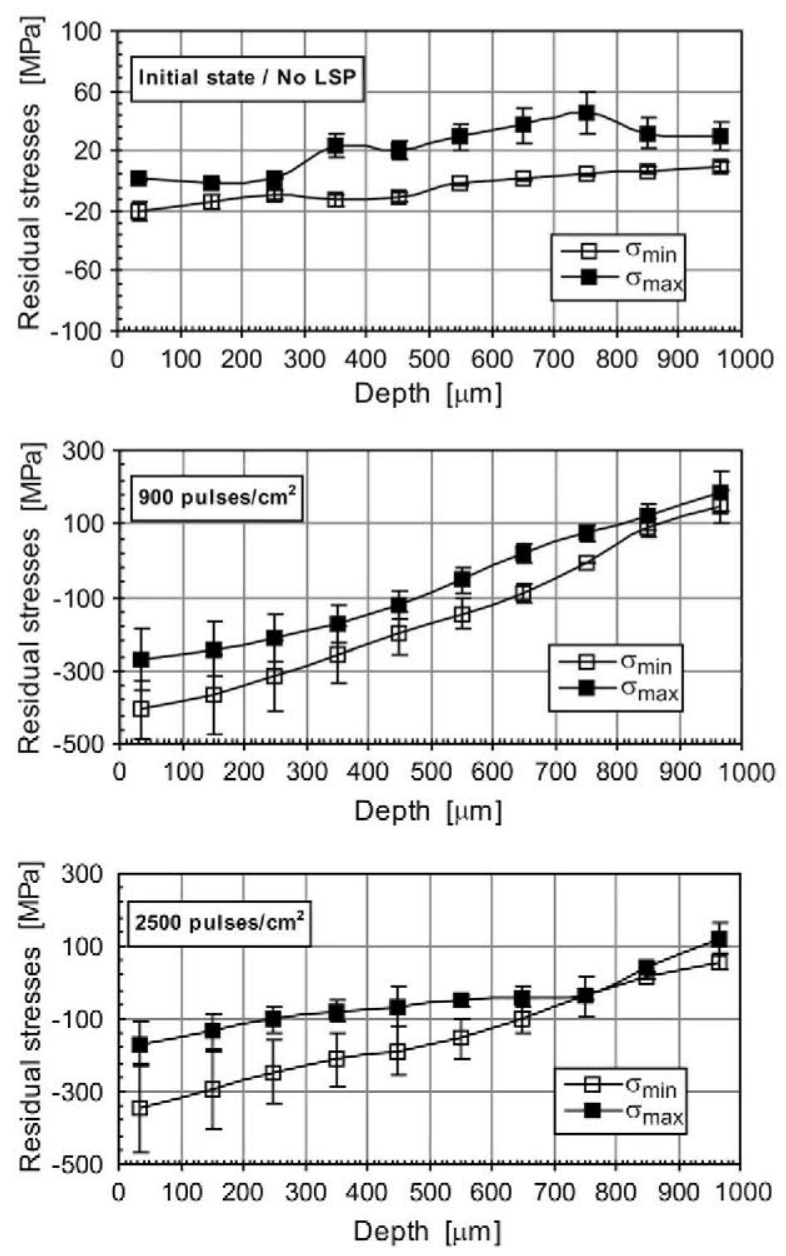

Fig. 9. Hole drilling principal residual stresses.

The analysis of the principal residual stress (RS) profiles after laser shock peening for both pulse densities are predominantly compressive. In the case of LSPwC treatment with 900 pulses $/ \mathrm{cm}^{2}, \sigma_{\max }$ and $\sigma_{\min }$ changed to tensile state after reaching depths of approximately $620 \mu \mathrm{m}$ and $770 \mu \mathrm{m}$, respectively. Meanwhile, after LSPwC with 2500 pulses $/ \mathrm{cm}^{2} \sigma_{\max }$ and $\sigma_{\min }$ reached a tensile state at depths $810 \mu \mathrm{m}$ and $820 \mu \mathrm{m}$, respectively. Thus, higher strain hardening depth (higher compression) due to the purely mechanical effect of shock waves is obtained with a higher pulse density. However, the highest compressive RS at the near surface $(33 \mu \mathrm{m})$ were obtained

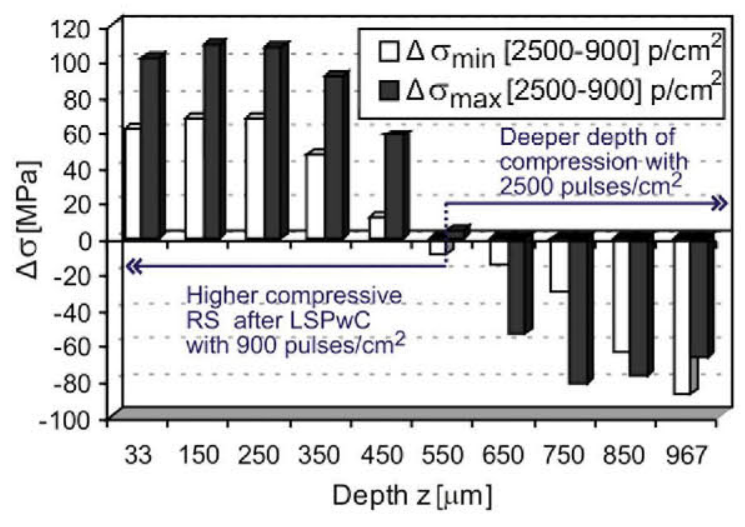

Fig. 10. Differences in principal residual stress profile. after 900 pulses $/ \mathrm{cm}^{2}$ in the transverse direction $\sigma_{\min }=-407 \pm$ $81 \mathrm{MPa}$, whereas after LSPWC with 2500 pulses $/ \mathrm{cm}^{2}$ RS of $-346 \pm$ $124 \mathrm{MPa}$ were obtained. Due to excessive shock wave pressure at specimens surface the highest compressive RS exceeded "quasi" static tensile strength $\left(\sigma_{m}\right)$ of the base material.

Furthermore, it is observed that higher near-surface compressive RS is achieved in the transverse direction of laser-beam passage, regardless of the pulse density. Similar RS results were obtained in our previous publications $[11,18,29]$ and are consistent results after LSP in other publication $[7,14,42]$.

Moreover, from the residual stress profile, a different gradient is observed in dependence of pulse density level, which changes more rapidly with lower pulse density.

In order to evaluate RS differences more precisely, residual stress difference of LSPWC specimen (2500-900 pulses $\left./ \mathrm{cm}^{2}\right)$ at specific depth is given in Fig. 10.

As demonstrated in Fig. 10, the highest compressive RS and the most distinctive difference of RS are obtained just below the surface (higher compression after 900 pulses $/ \mathrm{cm}^{2}$ ). Afterwards, a constant decrease is observed up to a depth of approximately $450 \mu \mathrm{m}$. Furthermore, higher compressive residual stresses in the specimen treated with 2500 pulses $/ \mathrm{cm}^{2}$ are observed from a depth of approximately $550 \mu \mathrm{m}$. Afterwards, almost a constant increase of compression is present with higher pulse density. Relaxation of compressive RS at the surface at higher pulse densities is probably associated with thermal effect relaxation due to the cumulative action of the plasma effect and shock waves on the thin surface layer. This explanation is also in accordance with micro-hardness distribution and reduction, which is present at higher pulse density (Fig. 7).

Micro-hardness and residual stress results obtained in our study are consistent with those reported by Sánchez-Santana et al. [15], Rubio-Gonzales et al. [41] and Sathyajith and Kalainathan [40] concerning the application of laser shock peening on aluminium alloys.

According to Sathyajith and Kalainathan [41], micro-hardness reduction at the surface is probably attributed to the increase of local/ surface thermal effect due to higher pulse density. Nevertheless, higher pulse density level shows smaller reduction of the residual stress through depth profile, which indicates higher strain hardening with Ref. [41]. A similar conclusion was reported by Clauer [2], where it was shown that plastically affected depth linearly increases with an increasing number of laser spot impacts. Thus, higher dislocation density is achieved.

\section{Conclusions}

It has been demonstrated that 3D topography measurements by Focus-Variation make it possible to characterise even strongly varying rough surfaces with high precision and resolution.

The 3D amplitude parameters, i.e. topography roughness, confirmed the overall trend; higher pulse density causes higher roughness due to the numerous cumulative actions of surface ablation and plasma and shock waves.

The auto-correlation model of surface roughness confirmed the strong preferential trend of the non-treated specimen's surface texture orientation in the cutting direction. Meanwhile, LSPWC's small tendency of the surface texture orientation is present, although it is more pronounced with higher pulse density level.

Analysis of LSPWC-induced surface craters indicated better surface condition after LSPWC treatment with 900 pulses $/ \mathrm{cm}^{2}$, i.e. similar surface condition in both ( $\mathrm{L}$ and $\mathrm{T}$ ) directions and smaller crater depth (peak-to-peak amplitude) compared to LSPwC with 2500 pulses $/ \mathrm{cm}^{2}$. Besides, after LSPWC treatment with 2500 pulses $/ \mathrm{cm}^{2}$ crater wave period is by the factor 4.3 larger compared to the specimen treated with lower pulse density. Furthermore, after 2500 pulses $/ \mathrm{cm}^{2}$, higher increases of the LSPwC-induced crater depth (peak-to-peak amplitude) 
and wave period were observed in the transverse direction. The crater area after 900 pulses $/ \mathrm{cm}^{2}$ was equal to $867.92 \mu \mathrm{m}^{2}$ in the $L$ direction, whereas in the $T$ direction, of $1088.12 \mu \mathrm{m}^{2}$ was achieved, which means a $220 \mu \mathrm{m}^{2}$ increase compared to the $L$ direction. In the case of higher pulse density ( 2500 pulses $/ \mathrm{cm}^{2}$ ), the largest crater area was obtained in the $T$ direction, with $1337.93 \mu^{2}$, indicating a $470 \mu^{2}$ increase compared to the specimen with the lowest value $\left(900\right.$ pulses $/ \mathrm{cm}^{2}$ and $L$ direction).

The micro-hardness depth profile confirmed an increase after LSPWC at both pulse densities, whereas LSPWC-generated shock waves showed an impact of up to $800 \mu \mathrm{m}$ below the surface. Thus, it has been shown that the LSPWC process improves the mechanical properties of AA6082-T651 aluminium alloy.

Residual stress evaluation confirmed laser shock peening as an effective method of inducing compressive residual stresses in the near surface layer of investigated aluminium alloy. Furthermore, it has been shown that higher compressive residual stresses are achieved in the transverse direction of laser-beam movement, i.e. normal to the LSPWC scan direction.

\section{References}

[1] B.P. Fairand, A.H. Clauer, in: AIP Conference Proceedings, 50, 1978, p. 27.

[2] A.H. Clauer, in: J.K. Gregory, H.J. Rack, D. Eylon (Eds.), Surface Performance of Titanium, TMS, Warrendale, 1996, p. 217.

[3] U. Trdan, J. Grum, M.R. Hill, Mater. Sci. Forum 681 (2011) 480.

[4] P. Peyre, X. Scherpereel, L. Berthe, C. Carboni, R. Fabbro, G. Béranger, C. Lemaitre, Mater. Sci. Eng. A280 (2000) 294.

[5] G. Hammersley, L.A. Hackel, F. Harris, Opt. Lasers Eng. 34 (2000) 327.

[6] K. Ding, L. Ye, Laser Shock Peening: Performance and Process Simulation, First ed. Woodhead Publishing, Cambridge, 2006.

[7] E. Maawada, Y. Sano, L. Wagner, H.-G. Brokmeier, Ch. Genzel, Mater. Sci. Eng., A $536(2012) 82$.

[8] N. Mukai, N. Aoki, M. Obata, A. Ito, Y. Sano, C. Konagai, in: Proceedings of the Third JSME/ASME International Conference on Nuclear Engineering (ICONE-3), Kyoto, 1995 , p. 1489.

[9] G. Ivetic, I. Meneghin, E. Troiani, G. Molinari, J.L. Ocaña, M. Morales, J. Porro, A. Lanciotti, V. Ristori, C. Polese, J. Plaisier, A. Lausi, Mater. Sci. Eng., A 534 (2012) 573.

[10] P. Peyre, R. Fabbro, P. Merrien, H.P. Lieurade, Mater. Sci. Eng. A210 (1996) 102.

[11] U. Trdan, J.L. Ocaña, J. Grum, J. Mech. Eng. $57 / 5$ (2011) 385, http://dx.doi.org/ $10.5545 /$ svjme.2010.119.
[12] Y. Sano, K. Alkita, K. Takeda, R. Sumiya, T. Tazawa, T. Saito, C. Narazaki, Int. J. Struct. Integr. 2 (2011) 42.

[13] Z. Hong, Y. Chengye, Mater. Sci. Eng. A257 (1998) 322

[14] Y. Sano, M. Obata, T. Kubo, N. Mukai, M. Yoda, K. Masaki, Y. Ochi, Mater. Sci. Eng. A417 (2006) 334.

[15] U. Sánchez-Santana, C. Rubio-González, G. Gomez-Rosas, J.L. Ocaña, C. Molpeceres, J. Porro, M. Morales, Wear 260 (2006) 847.

[16] U. Trdan, S. Žagar, J. Grum, J.L. Ocaña, Int. J. Struct. Integr. 2 (2011) 9.

[17] Y. Hu, Z. Yao, Int. J. Mach. Tools Manuf. 48 (2008) 152.

[18] U. Trdan, J. Grum, Corros. Sci. 59 (2012) 324.

[19] C. Vargel, Corrosion of Aluminium, Elsevier Science, San Diego, USA, 2004.

[20] C.N. Panagopoulos, E.P. Georgiou, A.G. Gavras, Tribol. Int. 42 (2009) 886.

[21] O. Hatamleh, J. Smith, D. Cohen, R. Bradley, Appl. Surf. Sci. 255 (2009) 7414.

[22] F.Z. Dai, J.Z. Lu, Y.K. Zhang, K.Y. Luo, Q.W. Wang, L. Zhang, X.J. Hua, Vacuum 86 (2012) 1482.

[23] J.Z. Lu, K.Y. Luo, Y.K. Zhang, C.Y. Cui, G.F. Sun, J.Z. Zhou, L. Zhang, J. You, K.M. Chen, J.W. Zhong, Acta Mater. 58 (2010) 3984

[24] W.P. Dong, K.J. Stout, Wear 181-183 (1995) 700

[25] A.T. Nguyen, D.L. Butler, Meas. Sci. Technol. 16 (2005) 1813.

[26] T.Y. Lin, S.H. Lu, K.J. Stout, Int. J. Mach. Tools Manuf. 35 (2) (1995) 239.

[27] K.J. Stout, L. Blunt, Surf. Coat. Technol. 71 (1995) 69.

[28] K.H. Wang, L. Blunt, L.T. Mao, C.C. Chao, K.J. Stout, Int. J. Mach. Tools Manuf. 41 (2001) 2071.

[29] G. Gomez-Rosasa, C. Rubio-Gonzalez, J.L. Ocaña, C. Molpeceres, JA. Porroc, M. Morales, F.J. Casillas, Appl. Surf. Sci. 256 (2010) 5828.

[30] IFM Manual, IFM G4 3.2 Demo EN 29.04.2009, Alicona GmbH.

[31] R. Danzl, F. Helmli, S. Scherer, in: Proc. 11th Int. Conf. on Metrology and Properties of Engineering Surfaces, 2007, p. 41.

[32] ISO 25178-6, Geometrical product specifications (GPS) Part 6: Classification of methods for measuring surface texture, Draft, 2010.

[33] C. Courbon, D. Kramar, P. Krajnik, F. Pušavec, J. Rech, J. Kopač, Int. J. Mach. Tools Manuf. 49 (2009) 1114.

[34] ASTM Standard 'Standard Test Method for Determining Residual Stress by the Hole-Drilling, ASTM E 837-08, ASTM Int., USA, 2008.

[35] Gary S. Schajer, H-Drill User Manual, Version 3.10, 2000-2007.

[36] P.V. Grant, J.D. Lord, MATC (MN)31, National Physical Laboratory, UK, 2002.

[37] W.P. Dong, E.J. Davis, D.L. Butler, K.J. Stout, Tribol. Int. 28/7 (1995) 453.

[38] D.C. Montgomery, Design and Analysis of Experiments, John Wiley \& Sons, New York, 2001.

[39] J. Grum, T. Kek, Thin Solid Films 453-454 (2004) 94.

[40] S. Sathyajith, S. Kalainathan, Opt. Lasers Eng. 50 (2012) 345.

[41] C. Rubio-Gonzalez, J.L. Ocaña, G. Gomez-Rosas, C. Molpeceres, M. Paredes, A. Banderas, J. Porto, M. Morales, Mater. Sci. Eng.. A 386 (2004) 291.

[42] E. Maawad, H.-G. Brokmeier, L. Wagner, Y. Sano, Ch. Genzel, Surf. Coat. Technol. 205 (2011) 3644. 